\section{Percepção e expressão de sintomas depressivos em três grupos culturais catarinenses: açorianos, italianos e alemães}

\author{
Perceptions of the symptoms of depression between members of three \\ ethnic communities in Santa Catarina: Azoreans, Italians and Germans
}

Jaime Lin', Mirella M. Peruchi', Luciana Hammes de Souza', Leticia M. Furlanetto', Esther Jean Langdon’

\section{RESUMO}

Objetivos: Comparar a percepção da depressão, incluindo a percepção dos sintomas e dos tratamentos considerados apropriados, pelos membros de três grupos étnicos (descendentes de açorianos, italianos e alemães residentes em Santa Catarina, Estado da Região Sul do Brasil), assim como a expressão de depressão por meio do Inventário Beck de Depressão nestes grupos. Metódo: Em um estudo transversal, com uma parte quantitativa e uma qualitativa, foram avaliados 60 indivíduos, sendo 20 de cada uma das comunidades étnicas escolhidas: açoriana, italiana e alemã. Realizou-se uma entrevista semi-aberta, tendo como questões orientadoras: Para você, o que é depressão?; O que pessoas deprimidas devem fazer?; e Qual atividade de lazer você mais gosta de fazer? Depois, foi aplicado o Inventário Beck de Depressão (BDI). Os escores do BDI (subescalas: cognitiva-afetiva e somática) foram comparados entre os diferentes grupos por meio do método ANOVA. No estudo qualitativo, verificaram-se as percepções compartilhadas no discurso dos indivíduos de cada grupo, quanto à depressão e seu tratamento. Resultados: Os principais sintomas relatados por descendência foram: irritabilidade (açorianos), autopunição (italianos) e falta de energia (alemães). Não houve diferença estatisticamente significativa quanto à freqüência de sintomas cognitivos e somáticos nas diferentes comunidades. O padrão de percepção da depressão caracterizou-se nos descendentes de açorianos por isolamento ("... a gente não quer ver ninguém"). Nos italianos, a depressão esteve relacionada a afastamento da família ("Depressão é vontade até de deixar o filho, o marido"), sendo esta vista como a responsável por ajudar na melhora. Já entre os alemães, a depressão esteve relacionada à dificuldade no trabalho ("Depressão é... não ter mais vontade de trabalhar") e este foi relatado como a solução. Conclusão: De acordo com o grupo étnico, a expressão, a percepção e a busca de tratamento para a depressão relacionou-se: à comunidade (açoriana), à família (italiana) e ao trabalho (alemã).

\section{ABSTRACT}

Objectives: To compare perceptions of the symptoms of depression and its management between members of three ethnic communities (descendants of Azoreans, Italians and Germans in

1 Departamento de Clínica Médica do Centro de Ciências da Saúde da Universidade Federal de Santa Catarina (UFSC); Departamento de Antropologia do Centro de Filosofia e Ciências Humanas da UFSC. 


\section{Key-words}

Depression, perception, culture, ethnicity, diagnosis.
Santa Catarina, which is a State in the South of Brazil) and to compare the expression of depressive symptoms via the Beck Depression Inventory. Methods: In a cross-sectional study, with both a quantitative and a qualitative design, 60 individuals (20 of each of the above ethnicities) were interviewed. A semi-structured interview was conducted, oriented by the following questions: In your opinion, what is depression?; How should persons cope with depression? and What is your preferred leisure time activity? After that, the subjects completed the Beck Depression Inventory $(B D I)$. The BDI scores (subscales: cognitive/affective and somatic) were compared among the three groups using ANOVA. In the qualitative study, patterns of perceptions concerning depression were identified analyzing the responses of the individuals to the semi-structured interviews. Results: The most frequent self-reported symptoms according to each ethnicity were: irritability (Azoreans), self-punishment (Italians) and low energy (Germans). There were no statistically significant differences between cognitive or somatic scores among the groups. The Azorean descendants suspected depression if the individuals evidenced social withdrawal ("... we don't want to see anybody"). For the Italian descendants, the family was cited as important in detecting ("Depression is feeling like leaving your son, your husband") and managing depression. The German descendants attributed depression to difficulty with work ("Depression is... not wanting to work") and work was also seen as the solution. Conclusions: According to ethnicity, the detection, expression of symptoms and search for treatment was related to: the community (Azoreans); the family (Italians) and work (Germans).

\section{INTRODUÇÃO}

A depressão é atualmente reconhecida um problema prioritário de saúde pública, uma vez que vem sendo associada ao aparecimento posterior de diversas doenças físicas ${ }^{1-3}$, além de contribuir para a piora na adesão, na evolução e na qualidade de vida de pacientes clínicos ${ }^{4-8}$. Apesar disso, menos da metade dos indivíduos com esta doença recebem tratamento antidepressivo $0^{9,10}$. O contexto cultural dos indivíduos é um dos fatores que modula este problema, já que a expressão dos sintomas depressivos, o reconhecimento de que seja doença e a atitude considerada adequada, quando esta é detectada, variam de acordo com a etnia dos indivíduos $^{11}$. Nesse sentido, a psiquiatria transcultural vem tentando descrever o impacto que as diferenças socioculturais exercem sobre os transtornos mentais e seu tratamento ${ }^{12-14}$. Apesar disso, a padronização dos critérios diagnósticos das doenças mentais e os instrumentos de avaliação ainda estão longe de abarcar a complexidade da influência cultural na determinação dos fenômenos mentais ${ }^{15}$. Por isso, o conhecimento da percepção sobre o que seja a doença depressão e seu melhor manejo em um grupo étnico específico pode ajudar no reconhecimento e no tratamento precoce desses pacientes.

A maior parte dos estudos comparativos entre culturas, abrangendo os aspectos da depressão, é feita comparando-se culturas ocidentais e orientais, que obviamente apresentam grandes diferenças na expressão de seus sentimentos. Uma dessas diferenças, talvez a mais importante e já demonstrada por vários estudos ${ }^{16-20}$, é a predominância de sintomas somáticos em indivíduos de culturas orientais, enquanto os ocidentais a expressariam por meio de sintomas psíquicos e cognitivos. Entretanto, ainda há controvérsias neste sentido. Uma pesquisa realizada em 14 países revelou que quando os médicos generalistas perguntavam para pacientes deprimidos com queixas somáticas sobre sintomas cognitivo-afetivos estes admitiam, e que o que influenciava na forma de expressão era a maneira como eram feitas as consultas: personalizadas ou não ${ }^{21}$. Assim, a queixa somática serviria como um "ticket de admissão" para o paciente deprimido no sistema de saúde ${ }^{21}$.

No Brasil, são poucos os estudos nessa área. Após consulta à base de dados Lilacs entre os anos de 1985 a 2007, usando os descritores "depressão" ou "sintomas depressivos" e "cultura" ou "transcultural", foram encontrados poucos estudos que enfocam a psiquiatria transcultural, visto que nenhum deles comparou a percepção ou a expressão de sintomas depressivos em grupos de origens culturais diferentes.

Esse déficit de trabalhos transculturais enfocando a depressão no Brasil talvez se justifique, considerando a grande diversidade cultural encontrada no Brasil hoje. Sabe-se que não há culturas "puras", mas também que as fronteiras não são claras entre um grupo étnico e outro. Porém, aqui utilizamos o conceito de cultura a fim de se referir ao sistema simbólico compartilhado por um grupo que influencia na percepção, na interpretação e na ação dos indivíduos ${ }^{22}$. Esse conceito, inspirado por Geertz $(1978)^{23}$, reconhece que os processos de saúde e doença são altamente influenciados pelo contexto sociocultural ${ }^{24}$ e o que a pessoa reconhece como doença, como interpreta os sintomas e a ação 
tomada para resolvê-la são resultados deste contexto. Assim, apesar de os vários grupos étnicos no Brasil não serem isolados uns dos outros, cada um tem suas características próprias, que necessitam ser estudadas no tocante à percepção e à expressão das doenças mentais, e que têm um impacto na maneira de perceber estados depressivos e decidir quais ações devem ser tomadas.

Os objetivos deste trabalho foram: descrever a freqüência dos diferentes sintomas depressivos em descendentes de açorianos, italianos e alemães residentes em Santa Catarina, analisando as semelhanças e diferenças dos sintomas depressivos auto-relatados, de acordo com o Inventário Beck de Depressão ${ }^{25}$ nos três grupos étnicos, e investigar as diferenças entre os grupos na percepção da depressão, dos sintomas associados à depressão e as interpretações de como melhor lidar com o problema.

\section{MÉTODOS}

Trata-se de um estudo transversal com uma parte quantitativa e outra qualitativa. Três comunidades do Estado de Santa Catarina, caracterizadas como expressivas da cultura dos imigrantes originais, foram escolhidas: Ribeirão da llha (Florianópolis), representando a imigração açoriana, o município de Nova Trento, representando a italiana, e o município de São Pedro de Alcântara, representando a alemã. A amostra foi composta de 20 indivíduos adultos de cada comunidade, totalizando 60 .

Os indivíduos foram selecionados nas respectivas comunidades, em suas casas, de acordo com sua disponibilidade e a sua ascendência, identificada pelo sobrenome, atentando para o fato de que em mulheres casadas a confirmação da ascendência foi feita pelo sobrenome de solteira. O número de indivíduos foi selecionado com base nos critérios de pesquisa qualitativa de Minayo ${ }^{26,27}$. Foram excluídos os indivíduos com idade inferior a 18 anos, os que não tinham fluência na língua portuguesa e os que se recusaram a participar.

Foram colhidos dados sociodemográficos e de história psiquiátrica. A história pessoal e familiar foi feita de acordo com o modelo realizado por Koenig ${ }^{28}$. Além disso, foi feita entrevista semi-aberta para avaliar a percepção da depressão, formulada pelos autores. Foram feitas perguntas orientadoras a todos os indivíduos, além de outras que posteriormente julgou-se necessário. As perguntas orientadoras foram: "Para você, o que é depressão?"; "O que uma pessoa deprimida deve fazer?"; e "Qual atividade de lazer você mais gosta de fazer?". As entrevistas foram gravadas em fita cassete para posterior transcrição e análise qualitativa. Depois da entrevista aberta, foi aplicada a versão validada em português do Inventário Beck de Depressão (BDI) ${ }^{29}$ para avaliar os sintomas depressivos. Foram registradas as reações das pessoas às perguntas da entrevista.
A análise estatística foi feita utilizando-se o programa SPSS, versão 10.0 para Windows ${ }^{30}$. Foram descritas as porcentagens, as médias e os desvios-padrão, conforme apropriado tanto da amostra total quanto separadamente para cada uma das três diferentes culturas. Os sintomas foram considerados presentes quando o escore naquele item era igual ou superior a dois. Foram avaliados como parâmetros a idade, o gênero, a história psiquiátrica pessoal e a história psiquiátrica familiar e descritas as porcentagens, médias e desvios-padrão, conforme apropriado.

As amostras referentes a cada um dos respectivos grupos étnicos (açorianos, italianos e alemães) foram comparadas quanto a idade, escolaridade, renda, história psiquiátrica pessoal e história psiquiátrica prévia, a fim de se verificar possíveis diferenças estatísticas entre os grupos.

Dividiu-se o BDI em duas subescalas, sendo uma de sintomas cognitivo-afetivos (primeiros 13 itens) e outra de sintomas somáticos (do item 14 ao 21), permitindo a comparação da freqüência de sintomas cognitivo-afetivos e sintomas somáticos pelo método ANOVA dos escores obtidos nas três culturas.

Foram considerados estatisticamente significativos valores de $\mathrm{p}<0,05$.

O conteúdo das entrevistas foi analisado para identificar as semelhanças e as diferenças das representações e percepções expressadas pelos entrevistados.

Esta pesquisa foi aprovada pelo Comitê de Ética em Pesquisa em Seres Humanos da Universidade Federal de Santa Catarina (UFSC), e todos os indivíduos assinaram termo de consentimento após esclarecimento sobre o estudo.

\section{RESULTADOS}

Houve predomínio do sexo feminino (83,3\%), com média de idade \pm desvio-padrão (DP) de 54,3 $\pm 15,7$ anos. A maioria dos participantes possuía escolaridade média \pm DP de $5,6 \pm 4,1$ anos, era casada ou mantinha união estável (76,7\%) e possuía renda familiar de até $\mathrm{R} \$ 600,00$ (cerca de 300 dólares americanos) (53,3\%). A maioria dos participantes referiu ser "do lar", porquanto 48,3\% recebiam aposentadoria. Quanto à ascendência, 51,7\% dos participantes pertenciam da segunda à quarta geração nascida no Brasil (Tabela 1).

Em relação à história psiquiátrica prévia, 33,3\% dos entrevistados apresentaram história psiquiátrica pessoal prévia positiva e 45\% apresentaram história psiquiátrica familiar positiva.

De acordo com o BDI, 11,7\% dos 60 participantes do estudo apresentaram escores $>16$ no BDl, sugerindo síndrome depressiva com significado clínico. Desses, a maioria era do sexo feminino $(85,7 \%)$, com média de idade \pm desvio-padrão de 58,71 $\pm 21,15$ anos com história psiquiátrica prévia positiva em $71,4 \%$ e história psiquiátrica familiar positiva em $57,1 \%$. 
Tabela 1. Características sociodemográficas da amostra $(n=60)$

\begin{tabular}{|c|c|c|}
\hline Características & $n(60)$ & $\%$ \\
\hline \multicolumn{3}{|l|}{ Sexo } \\
\hline Masculino & 10 & 16,7 \\
\hline Feminino & 50 & 87,3 \\
\hline \multicolumn{3}{|l|}{ Idade (anos) } \\
\hline 18 a 39 & 14 & 23,3 \\
\hline 40 a 64 & 28 & 46,7 \\
\hline$\geq 65$ & 18 & 30,0 \\
\hline \multicolumn{3}{|l|}{ Estado civil } \\
\hline Solteiro(a) & 09 & 15,0 \\
\hline Casado(a)/união estável & 46 & 76,7 \\
\hline Separado(a) & 00 & 0,0 \\
\hline Viúvo(a) & 05 & 8,3 \\
\hline \multicolumn{3}{|l|}{ Geração no Brasil } \\
\hline $2^{\mathrm{a}} \mathrm{a} 4^{\mathrm{a}}$ & 30 & 51,7 \\
\hline $5^{\mathrm{a}} \mathrm{a} 8^{\mathrm{a}}$ & 06 & 10,4 \\
\hline Não sabe & 24 & 37,8 \\
\hline \multicolumn{3}{|l|}{ Escolaridade (anos) } \\
\hline 0 & 00 & 0,0 \\
\hline $1 \mathrm{a} 4$ & 42 & 70,0 \\
\hline 5 a 8 & 06 & 15,0 \\
\hline$>8$ & 12 & 20,0 \\
\hline \multicolumn{3}{|l|}{ Renda familiar } \\
\hline Até $R \$ 600,00$ & 33 & 55,0 \\
\hline $\mathrm{R} \$ 600,00$ a $R \$ 1.200,00$ & 16 & 26,7 \\
\hline Superior a $R \$ 1.200,00$ & 11 & 18,3 \\
\hline
\end{tabular}

Os sintomas mais freqüentes na amostra total foram: auto-acusação (26,7\%), irritabilidade (25\%) e falta de energia (17\%) (Tabela 2).

Os principais sintomas referidos de acordo com o grupo étnico foram: irritabilidade nos açorianos (30\%), autoacusação nos italianos (45\%), e falta de energia nos alemães (25\%).

Houve diferença estatisticamente significativa entre os três diferentes grupos (açoriano, italiano e alemão) quanto à idade $(p=0,01)$, sendo os indivíduos pertencentes à comunidade italiana mais jovens. Não houve diferença estatisticamente significativa quanto a escolaridade $(p=0,12)$, renda familiar ( $p=0,53)$, história psiquiátrica pessoal $(p=0,75)$ e história psiquiátrica familiar $(p=0,25)$ (Tabela 3).

Quando os escores obtidos no BDI foram divididos em subescalas cognitivo-afetiva e somática, verificaramse que os indivíduos das comunidades açoriana e italiana apresentavam maior freqüência de sintomas cognitivoafetivos, enquanto os indivíduos de descendência alemã apresentavam maior freqüência de sintomas somáticos.
Tabela 2. Freqüência de sintomas depressivos na amostra total $(n=60)$

\begin{tabular}{|c|c|c|c|c|}
\hline \multirow{2}{*}{ Sintomas } & \multicolumn{2}{|c|}{ Não* } & \multicolumn{2}{|c|}{$\operatorname{Sim}^{*}$} \\
\hline & $n$ & $\%$ & $n$ & $\%$ \\
\hline Tristeza & 56 & 93,3 & 4 & 6,7 \\
\hline Desesperança & 51 & 85,0 & 9 & 15,0 \\
\hline Sensação de fracasso & 57 & 95,0 & 3 & 5,0 \\
\hline Anedonia & 55 & 91,7 & 5 & 8,3 \\
\hline Culpa & 58 & 96,7 & 2 & 3,3 \\
\hline Sentimento de punição & 52 & 86,7 & 8 & 13,3 \\
\hline Desgosto consigo & 57 & 95,0 & 3 & 5,0 \\
\hline Auto-acusação & 44 & 73,3 & 16 & 26,7 \\
\hline Ideação suicida & 60 & 100,0 & 0 & 0,0 \\
\hline Choro fácil & 53 & 88,3 & 7 & 11,7 \\
\hline Irritabilidade & 45 & 75,0 & 15 & 25,0 \\
\hline Perda do interesse nas pessoas & 58 & 96,7 & 2 & 3,3 \\
\hline Indecisão & 52 & 86,7 & 8 & 13,3 \\
\hline Mudança na imagem do corpo & 51 & 85,0 & 9 & 15,0 \\
\hline Dificuldade no trabalho & 54 & 90,0 & 6 & 10,0 \\
\hline Insônia & 52 & 86,7 & 8 & 13,3 \\
\hline Falta de energia & 50 & 83,3 & 10 & 16,7 \\
\hline Falta de apetite & 53 & 88,3 & 7 & 11,7 \\
\hline Perda de peso & 56 & 93,3 & 4 & 6,7 \\
\hline Preocupação somática & 53 & 88,3 & 7 & 11,7 \\
\hline Perda de libido & 51 & 85,0 & 9 & 15,0 \\
\hline
\end{tabular}

*0s itens do Inventário Beck de Depressão foram categorizados em: Não = 0 (ausente) e 1 (leve); Sim = 2 (moderado) e 3 (grave).

Tais diferenças, no entanto, não foram estatisticamente significativas (Tabela 4).

Na comunidade açoriana a depressão foi freqüentemente relacionada a ficar remoendo fatos ruins (por exemplo, problemas financeiros) ou ao isolamento, como se pode notar nas frases a seguir:

"Depressão é uma coisa que o camarada encasqueta na cabeça e fica com a cuca como um balaio, como pegar um problema de dívida ou de família."

"Fica dando nos nervos, problemas financeiros, de família. É um estado de nervo que a pessoa tem. Se não se incomoda não tem. A pessoa nervosa cisma com algo e tem depressão."

"É horrivel né? A gente não tem vontade de nada, só chora, fica trancada no quarto, tem vontade de morrer, não quer ver ninguém..."

Tabela 3. Dados sociodemográficos e de história psiquiátrica comparativa entre as três culturas

\begin{tabular}{|c|c|c|c|c|c|}
\hline Caracteristicas & Açorianos & Italianos & Alemães & $\mathbf{F}$ & $p$ \\
\hline Idade: média ( \pm DP) anos & $58,05 \pm 12,65$ & $45,85 \pm 16,02$ & $58,90 \pm 15,28$ & 4,92 & $0,01^{*}$ \\
\hline Escolaridade: média ( $\pm D P$ ) anos & $6,55 \pm 5,11$ & $6,10 \pm 4,29$ & $4,05 \pm 2,06$ & 2,18 & 0,12 \\
\hline Renda: (\%) categorias $\mathrm{I} / \mathrm{II} / \mathrm{II} \mathrm{I}^{* *}$ & $25 / 50 / 25$ & $70 / 25 / 05$ & $70 / 05 / 25$ & 3,09 & 0,053 \\
\hline \multicolumn{6}{|l|}{ História de doença psiquiátrica } \\
\hline Pessoal (\%) & 40 & 30 & 30 & 0,29 & 0,75 \\
\hline Familiar (\%) & 60 & 40 & 35 & 1,41 & 0,25 \\
\hline
\end{tabular}

${ }^{*} p<0,05 ; *$ * Renda familiar: $I=$ até $R \$ 600,00 ; \|=$ entre $R \$ 600,00$ e $R \$ 1.200,00 ;$ e $|l|=>R \$ 1.200,00$. 
Tabela 4. Comparação entre sintomas cognitivo-afetivos e somáticos entre as três culturas

\begin{tabular}{lccccc}
\hline Características & Açorianos & Italianos & Alemães & $\mathbf{F}$ & $\mathbf{p}^{*}$ \\
\hline $\begin{array}{l}\text { Sintomas cognitivos: } \\
\text { média } \pm \text { DP }\end{array}$ & $6,95 \pm 6,92$ & $6,00 \pm 4,22$ & $4,40 \pm 5,07$ & 1,09 & 0,34 \\
$\begin{array}{l}\text { Sintomas somáticos: } \\
\text { média } \pm \text { DP }\end{array}$ & $4,90 \pm 3,87$ & $4,50 \pm 3,87$ & $5,10 \pm 3,17$ & 0,13 & 0,87 \\
& & & & &
\end{tabular}

*ANOVA.

A família, na comunidade italiana, revelou-se como a principal fonte de preocupações e muito importante para detectar a depressão, já que seus membros se unem nos momentos de lazer e aqueles que estão deprimidos tendem a não querer ver seus familiares, como se pode observar nas frases adiante:

"[Depressão] é quando alguma coisa ruim acontece e fica deixando a gente ruim também. Quando se perde alguém da família. A coisa mais triste é perder um filho porque vem do coração da gente."

"Depressão é vontade até de deixar o filho, o marido."

Quanto ao que deve ser feito quando alguém está deprimido, os descendentes de italianos referiram:

"Procurar a família antes. Muitas pessoas ficam falando em médico, mas às vezes é a família que ajuda..."

"[A pessoa] Deve pensar em coisas boas, ouvir música, sair, se distrair, mas deve procurar a família sempre, depois a família decide qual é o melhor tratamento".

Finalmente, entre os descendentes de alemães houve enorme dificuldade em se definir o conceito de depressão, como pode ser observado nas frases a seguir:

"Depressão, não sei. Antigamente nem se falava, isso foi os médicos que arranjaram, sei lá o que é isso."

"Depressão, não sei o que é. Talvez pressão alta ou nos nervos."

O principal sintoma referido pelos descendentes de alemães foi a falta de energia, sendo facilmente correlacionado com os padrões de respostas encontrados, em que o trabalho exerce grande valor positivo na comunidade estudada, figurando entre as atividades mais importantes, incluindo, também, as de lazer, e como a principal forma de se lidar com pensamentos ruins, como pode ser observado a seguir:

"Depressão é não querer fazer nada, é um não consigo, é perder a vontade para as coisas, não ter mais vontade de trabalhar."

"A melhor coisa é tirar da cabeça. Enquanto eu puder dizer que é passageiro eu digo... tem que ter força. Trabalhar faz bem para tudo. Se eu parasse e ficasse vivendo a vida, pedia para morrer. Trabalhar faz parte da saúde."

\section{DISCUSSÃO}

Comparando os três grupos étnicos, verificamos que existe maior freqüência nos descendentes de açorianos e italianos de sintomas cognitivo-afetivos do que nos descendentes de alemães, que relataram mais sintomas somáticos. Embora essa diferença não tenha sido estatisticamente significativa, nos mostra uma tendência. Diversos estudos referem que a presença de sintomas somáticos é maior em culturas nas quais existe grande dificuldade em se definir o sofrimento psicológico, muitas vezes substituindo-o por sintomas mais facilmente traduzíveis, como dor ou sintomas físicos ${ }^{17,21}$. A presença de sintomas somáticos é também mais prevalente entre culturas em que os transtornos psiquiátricos carregam em si um grande estigma ${ }^{21}$. Nesses casos, o sofrimento psíquico acaba por ser ignorado, escondido ou somatizado ${ }^{18}$. Sintomas cognitivo-afetivos seriam, então, mais prevalentes naquelas culturas em que existe menos inibição em referir sintomas afetivos ${ }^{31}$ e nas comunidades em que existe comunicação verbal mais aberta e a expressão direta do sofrimento psíquico é mais bem aceita ${ }^{20,32}$. É possível, também, que por termos entrevistado as pessoas em suas casas e sem caracterizar como uma consulta médica, os indivíduos (mesmo os alemães) tenham admitido mais sintomas afetivos do que fariam se fossem procurar atendimento, o que estaria de acordo com Simon et al. ${ }^{21}$, que descreveram que a expressão da depressão por meio de sintomas somáticos seria mais freqüente quando o tipo de atendimento não era personalizado e o paciente tinha de provar que tinha uma doença física para receber atenção do médico²1.

Individualmente cada um dos grupos apresentou diferenças na expressão de sintomas depressivos, porquanto essas diferenças foram salientadas quando se uniram aos dados quantitativos com as respostas obtidas nas entrevistas qualitativas. O cruzamento dos dados permitiu caracterizar cada uma das culturas quanto à percepção e à expressão de sintomas depressivos.

$\mathrm{Na}$ comunidade açoriana, as relações interpessoais se revelaram de grande importância e, talvez, por isso a depressão seja detectada quando o indivíduo se isola da comunidade. Uma pesquisa realizada em outra comunidade nativa da llha de Florianópolis confirma nossos achados e demonstra a relação entre carências afetivas e a doença popular de "nervos"33. Nesse sentido, é interessante notar a importância do apoio social como proteção contra os efeitos de fatores estressores ${ }^{34,35}$.

Entre os descendentes de italianos, é possível que o fato de o sintoma auto-acusação ter sido o mais relatado tenha relação com a forma de catolicismo praticada pelo grupo. A religião pode influir na forma como a pessoa se vê no mundo ${ }^{36-38}$. A religiosidade do grupo torna-se ainda mais evidente quando se atenta para o fato de que Nova Trento, SC 
constitui local de turismo religioso - uma vez que é local de origem de Santa Paulina, canonizada em maio de 2002. Quanto à importância exercida pelo núcleo familiar, se por um lado ela pode ser fonte de sofrimento, atraso no diagnóstico da depressão ou ainda motivo de não tratamento de transtornos depressivos, por outro lado, pode ser importante fonte de suporte e apoio social. Estudos sugerem que ainda mais importante que a quantidade de interações sociais seria a qualidade dessas interações, exercendo papel protetor ainda maior diante de fontes de sofrimento ${ }^{34,35}$. Na amostra estudada, os fortes laços familiares poderiam ser um dos fatores responsáveis pela menor prevalência de depressão entre os descendentes de italianos.

A falta de energia citada como principal sintoma entre os descendentes de alemães poderia estar relacionada com a enorme importância que o trabalho e a capacidade de trabalhar exercem neste grupo. Tais dados já foram descritos previamente em trabalhos feitos em comunidades nas quais o valor de um indivíduo está na sua capacidade de produção e de trabalho17,21. Uma pesquisa da relação entre depressão e suicídio no contexto de uma comunidade coIona marcada pela cultura e linguagem alemã no Rio Grande do Sul também aponta o valor da capacidade individual de produção e de trabalho como fator importante nos processos socioculturais que impactam a saúde mental dos residentes ${ }^{39}$.

Algumas limitações, no entanto, foram encontradas durante a execução do trabalho. A coleta dos dados dependeu do auxílio das Secretarias de Saúde das respectivas cidades e comunidades e da disponibilidade de agentes comunitários que auxiliaram no acesso às comunidades. Por causa disso, a coleta de dados restringiu-se aos dias úteis e aos horários comerciais, o que influenciou na predominância de indivíduos do sexo feminino e de aposentados na amostra. Além disso, há de se ter cautela ao se descrever a freqüência de "depressão" e "sintomas depressivos", pois foi utilizado um instrumento, o BDI, para se definir a depressão. Como se sabe, o diagnóstico da depressão é clínico e depende do julgamento de um profissional experiente. As escalas foram elaboradas para auxiliar no rastreamento e em pesquisa. Apesar disso, vários trabalhos que relatam os efeitos deletérios, em virtude da presença de sintomas depressivos, utilizaram essa escala $a^{4,7}$. Outra limitação: por causa do tamanho da amostra e do uso de uma escala de depressão em uma amostra populacional, não é possível tirar conclusões em relação à psiquiatria transcultural sobre a presença de sintomas depressivos em grupos étnicos. Contudo, esta pesquisa gerou dados que podem ser testados no futuro, utilizando um desenho com essa finalidade.

Como significado clínico dos achados, ressaltamos que ainda existe enorme dificuldade em se definir depressão e, que, quando se consegue detectá-la, muitas vezes os indivíduos relutam em procurar auxílio médico, preferindo pro- curar auxílio na família, entre os membros da comunidade ou por meio do trabalho, retardando a melhora, podendo cronificar o quadro. Para minimizar esse problema, é importante que os profissionais de saúde estejam atentos, pois o atendimento médico na maioria das vezes é procurado em razão de outras queixas, que não por tristeza ou depressão. Assim, irritação, culpa e cansaço, mesmo que aparentemente justificados por fatores externos, podem ser a forma de expressão do sentimento de insuficiência da depressão, dependendo do contexto cultural do indivíduo.

\section{CONCLUSÕES}

Os sintomas mais freqüentes de acordo com a cultura foram: irritabilidade, na açoriana; auto-acusação, na italiana; e falta de energia, na alemã.

Houve diferenças na percepção da depressão, seus sintomas e tratamentos apropriados quando alguém está deprimido: na açoriana, o isolamento da comunidade e esta ajudando na melhora; na italiana, o isolamento da família e esta decidindo o que fazer; e na alemã, a falta ao trabalho e este como solução para depressão.

É necessária a realização de novos estudos com amostras maiores, testando as hipóteses levantadas nesta pesquisa. Estes resultados podem auxiliar no reconhecimento precoce e melhor abordagem de indivíduos deprimidos, de acordo com seus contextos socioculturais.

\section{AGRADECIMENTO}

O presente estudo contou com o apoio do CNPq, por meio de Bolsa de Iniciação Científica (PIBIC) concedida ao primeiro autor (Lin J).

\section{REFERÊNCIAS}

1. Engum A. The role of depression and anxiety in onset of diabetes in a large populationbased study. J Psychosom Res. 2007;62(1):31-8.

2. Everson $S A$, Kaplan GA, Goldberg DE, Salonen JT. Hypertension incidence is predicted by high levels of hopelessness in finnish men. Hypertension. 2000;35(2):561-7.

3. Larson SL, Owens PL, Ford D, Eaton W. Depressive disorder, dysthymia, and risk of stroke: thirteen-year follow-up from the Baltimore epidemiologic catchment area study. Stroke. 2001;32(9):1979-83.

4. Caulin-Glaser T, Maciejewski PK, Snow R, LaLonde M, Mazure C. Depressive symptoms and sex affect completion rates and clinical outcomes in cardiac rehabilitation. Prev Cardiol. 2007;10(1):15-21

5. Gross R, Olfson M, Gameroff MJ, Carasquillo 0, Shea S, Feder A, et al. Depression and glycemic control in hispanic primary care patients with diabetes. J Gen Intern Med. 2005;20(5):460-6.

6. Ng TP, Niti M, Tan WC, Cao Z, Ong KC, Eng P. Depressive symptoms and chronic obstructive pulmonary disease: effect on mortality, hospital readmission, symptom burden, functional status, and quality of life. Arch Intern Med. 2007;167(1):60-7. 
7. Rutledge T, Reis SE, Olson M, Owens J, Kelsey SF, Pepine (J, et al. Depression is associated with cardiac symptoms, mortality risk, and hospitalization among women with suspected coronary disease: the NHLBI-sponsored WISE study. Psychosom Med. 2006;68(2):217-23.

8. Sherwood A, Blumenthal JA, Trivedi R, Johnson KS, O'Connor CM, Adams KF, et al. Relationship of depression to death or hospitalization in patients with heart failure. Arch Intern Med. 2007;167(4):367-73

9. Cigognini MA, Furlanetto LM. Diagnosis and pharmacological treatment of depressive disorders in a general hospital. Rev Bras Psiquiatr. 2006;28(2):97-103.

10. Koenig HG. Depression outcome in inpatients with congestive heart failure. Arch Intern Med. 2006;166(9):991-6.

11. Helman CG, editor. Cultura, saúde e doença. 2a ed. Porto Alegre: Artes Médicas; 1994.

12. Beiser M. Culture and psychiatry, or "the tale of the hole and the cheese". Can J Psychiatry. 2003;48(3):143-4

13. Beiser M. Why should researchers care about culture? Can J Psychiatry. 2003;48(3):154-60.

14. Kirmayer $L J$, Minas H. The future of cultural psychiatry: an international perspective. Can J Psychiatry. 2000;45(5):438-46.

15. Jorge MR. Adaptação transcultural de instrumentos de pesquisa em saúde mental. In: Gorenstein C, Andrade LHSG, Zuardi AW, editores. Escalas de avaliação clínica em psiquiatria e psicofarmacologia. São Paulo: Lemos-Editorial. 2000; p. 53-7.

16. Chen IG, Roberts RE, Aday LA. Ethnicity and adolescent depression: the case of chinese americans. J Nerv Ment Dis. 1998;186(10):623-30.

17. Draguns JG, Tanaka-Matsumi J. Assessment of psychopathology across and within cultures: issues and findings. Behav Res Ther. 2003;41(7):755-76.

18. Furnham A, Malik R. Cross-cultural beliefs about "depression". Int J Soc Psychiatry. 1994;40(2):106-23.

19. Sartorius N. Cross-cultural research on depression. Psychopathology. 1986;19 Suppl 2:6-11.

20. Ulusahin A, Basoglu M, Paykel ES. A cross-cultural comparative study of depressive symptoms in British and Turkish clinical samples. Soc Psychiatry Psychiatr Epidemiol. 1994;29(1):31-9.

21. Simon GE, VonKorff M, Piccinelli M, Fullerton C, Ormel J. An international study of the relation between somatic symptoms and depression. N Engl J Med. 1999;341(18):1329-35.

22. Langdon, EJ. Cultura e os processos de saúde e doença. In: Jeolás LS, Oliveira M (Org.). Annais do Seminário Cultura, Saúde e Doença. Londrina: Ministério da Saúde; Universidade Estadual de Londrina; Secretaria Municipal de Ação Social/Prefeitura Municipal de Londrina, 2003. p. 91-107.
23. Geertz C. A interpretação das culturas. Rio de Janeiro: Zahar; 1978.

24. Young A. Some implications of medical beliefs and practices for social anthropology. Am Anthropologist. 1976;78(1):5-24.

25. Beck AT, Ward CH, Mendelson M, Mock J, Erbaugh J. An inventory for measuring depression. Arch Gen Psychiatry. 1961;4:561-71.

26. Minayo MCS. Pesquisa social: teoria, método e criatividade. 3a ed. Petrópolis: Vozes; 1994.

27. Minayo MCS. 0 desafio do conhecimento: pesquisa qualitativa em saúde. $6^{a}$ ed. Rio de Janeiro: Hucitec-Abrasco, 1999

28. Koenig HG, George LK, Meador KG. Use of antidepressants by nonpsychiatrists in the treatment of the medically ill hospitalized depressed elderly patients. Am J Psychiatry. 1997;154:1369-75

29. Gorenstein C, Andrade LHSG. Validation of a portuguese version of the beck depression inventory and the state-trait anxiety inventory in Brazilian subjects. Braz J Med Biol Res. 1996;29(4):453-7.

30. Norusis M. SPSS/PC 10.0 Base manual. Chicago: SPSS, Inc.; 2000.

31. Chang WC. A cross-cultural study of depressive symptomology. Cult Med Psychiatry. 1985:9(3):295-317

32. Becker AE, Kleinman A. Anthropology and psychiatry. In: Kaplan H, Saddock B, editores. Comprehensive textbook of psychiatry. Baltimore: Lippincott Williams \& Wilkins, 2000.

33. Silveira, ML. 0 nervo cala, o nervo fala: a linguagem da doença. Rio de Janeiro: Fio Cruz, 2000

34. Brugha TS, Weich S, Singleton N, Lewis G, Bebbington PE, Jenkins R, et al. Primary group size, social support, gender and future mental health status in a prospective study of people living in private households throughout Great Britain. Psychol Med. 2005;35(5):705-14

35. Blazer DG. Mood disorders: epidemiology. In: Sadock B, Sadock V, editores. Kaplan \& Sadock's comprehensive textbook of psychiatry. Baltimore: Lippincott Williams \& Wilkins; 2000. p. 3344

36. Cinnirella M, Loewenthal KM. Religious and ethnic group influences on beliefs about mental illness: a qualitative interview study. Br J Med Psychol. 1999;72(Dec;72):505-24.

37. Kleinman A. Rethinking psychiatry: from cultural category to personal experience. London: Free Press Collier Macmillan; 1988.

38. Kleinman A, Good B. Culture and depression: studies in the anthropology and cross-cultural psychiatry of affect and disorder. Berkeley: University of California Press; 1985

39. Heck, RM. Contexto sociocultural dos suicídios de colonos alemães: um estudo interdisciplinar para enfermagem [tese]. Santa Catarina: Universidade Federal de Santa Catarina, 2000. 